\title{
DEGREES OF CONSTANT-TO-ONE FACTOR MAPS
}

\author{
PAUL TROW
}

(Communicated by Thomas H. Brylawski)

\begin{abstract}
Let $f$ be a constant-to-one endomorphism of degree $d$, of a subshift of finite type $\Sigma_{A}$. If $p$ is a prime dividing $d$, then $p$ divides every nonleading coefficient of $\chi_{A}$, the characteristic polynomial for $A$. Further constraints are given for the possible degrees of a constant-to-one factor map between subshifts of finite type.
\end{abstract}

0. Introduction. It is well known that if $\Sigma_{A}$ and $\Sigma_{B}$ are irreducible subshifts of finite type of equal entropy, and $f: \Sigma_{A} \rightarrow \Sigma_{B}$ is a factor map (that is, a continuous, surjective map which commutes with the shift), then there is a positive integer $d$ such that $f$ is $d$-to-1 almost everywhere (i.e., every bilaterally transitive point of $\Sigma_{B}$ has exactly $d$ preimages under $f$ ). The integer $d$ is called the degree of $f$. The degree cannot be arbitrary: for a given $\Sigma_{A}$ and $\Sigma_{B}$, there are algebraic constraints on the possible degrees of factor maps $\Sigma_{A} \rightarrow \Sigma_{B}$. The first such constraint was. given by L. R. Welch [ 14.9 of $\mathbf{H}$ ], who showed that if $f$ is an endomorphism of the full shift on $n$ symbols, then the degree of $f$ divides a power of $n$. More recently, Boyle [B1] has shown that if $S$ and $T$ are sofic systems of entropy $\log \lambda$, then there exists a finite set $E$ of positive integers such that if $f: S \rightarrow T$ has degree $d$, then $d=e u$ where $e \in E$ and $u$ is a unit in $\mathbf{Z}[1 / \lambda]$. In this paper we give an analogous result for constant-to-one maps between subshifts of finite type. ( $f$ is constant-toone if every point has exactly $d$ preimages.) More specifically, we show that if $\Sigma_{A}$ and $\Sigma_{B}$ are subshifts of finite type and $\lambda$ is any nonzero eigenvalue for $A$ and $B$ (and $\lambda$ has the same multiplicity in $\chi_{A}$ and $\chi_{B}$ ), then there exists a finite set $E$ such that for any constant-to-one map $f: \Sigma_{A} \rightarrow \Sigma_{B}$ of degree $d$, we have $d=e u$, where $e \in E$ and $u$ is a unit in $\mathbf{Z}[1 / \lambda]$. As in Boyle's paper, this implies that if $f$ is an endomorphism, then in fact $d$ must be a unit in $\mathbf{Z}[1 / \lambda]$. It follows that if $p$ is a prime dividing $d$, then $p$ divides every nonleading coefficient of $\chi_{A}$.

In [B1], Boyle proves that if $f: S \rightarrow T(S, T$ sofic, of entropy $\log \lambda)$ has degree $d$, then the inverse image of certain cylinder sets breaks up into $d$ sets of equal measure, and so the measure of these sets is divisible by $d$ in $Z[1 / \lambda]$. In this paper, we show that under our more restrictive conditions, the inner product of a generalized left eigenvector and a right eigenvector for the matrix $A$ is divisible by $d$ in $\mathbf{Z}[1 / \lambda]$, for any nonzero eigenvalue $\lambda$. The remainder of the proof then follows exactly as in [B1].

I wish to thank M. Boyle, B. Kitchens and B. Marcus for helpful discussions concerning this paper.

Received by the editors August 29, 1986 and, in revised form, February 1, 1987. Presented December 1986, Conference on Coding Theory and Symbolic Dynamics, University of Maryland. 1980 Mathematics Subject ('lassification (1985 Revision). Primary 28D05.

Supported in part by a grant from the National Science Foundation. 
1. Background. We will assume some familiarity with subshifts of finite type and symbolic dynamics. For further background, see $[\mathbf{A}-\mathbf{M}]$ and $[\mathbf{P}-\mathbf{T}]$.

For the remainder of this paper, we will assume that $\Sigma_{A}$ and $\Sigma_{B}$ are irreducible subshifts of finite type, where $A$ and $B$ are nonnegative, integral matrices. Let $\mathscr{S}_{A}$ denote the symbol set of $\Sigma_{A}$. Thus $\mathscr{S}_{A}$ is the collection of edges in the directed graph for $A$ and $\Sigma_{A}=\left\{x \in \mathscr{S}_{A}^{\mathbf{Z}}: x_{i+1}\right.$ follows $x_{i}$ for all $\left.i\right\}$.

A one block map $f: \Sigma_{A} \rightarrow \Sigma_{B}$ is called right resolving if whenever $x_{1} \in \mathscr{S}_{A}$, $y_{1}, y_{2} \in \mathscr{S}_{B}, f\left(x_{1}\right)=y_{1}$ and $y_{1} y_{2}$ is allowed, then there exists a unique $x_{2} \in \mathscr{S}_{A}$ such that $f\left(x_{2}\right)=y_{2}$ and $x_{1} x_{2}$ is allowed. $f$ is left resolving if whenever $x_{2} \in \mathscr{S}_{A}$, $y_{1}, y_{2} \in \mathscr{S}_{B}, f\left(x_{2}\right)=y_{2}$ and $y_{1} y_{2}$ is allowed, then there exists a unique $x_{1} \in \mathscr{S}_{A}$ with $f\left(x_{1}\right)=y_{1}$ and $x_{1} x_{2}$ allowed.

A one block map $f: \Sigma_{A} \rightarrow \Sigma_{B}$ (i.e. a map on edges) defines a map $\hat{f}$ on vertices of the directed graph of $A$ as follows: if $i$ is a vertex and $e$ is any edge beginning at $i$, then $\hat{f}(i)$ is the initial vertex of $f(e)$. Clearly $\hat{f}$ is well defined.

If $A$ is $n \times n$ and $B$ is $k \times k$, then the relation matrix $F$ for $f$ is defined by

$$
R_{i j}= \begin{cases}1 & \text { if } \hat{f}(i)=j, \\ 0 & \text { otherwise }\end{cases}
$$

for $1 \leq i \leq n, 1 \leq j \leq k$. It is easy to check that if $f$ is right resolving then $A R=R B$, and if $f$ is left resolving then $R^{T} A=B R^{T}$ ( $T$ denotes transpose).

We will use the following theorem.

TheOREM (NASU [N, COROLlaRY 6.4]; ALSO IMPlicit IN [K]). Let $\Sigma_{A}$ and $\Sigma_{B}$ be irreducible subshifts of finite type, of equal entropy, and assume $f: \Sigma_{A} \rightarrow$ $\Sigma_{B}$ is constant-to-one. Then there exist topological conjugacies $g: \Sigma_{A} \rightarrow \Sigma_{\bar{A}}$ and $h: \Sigma_{B} \rightarrow \Sigma_{\bar{B}}$, and a one-block map $\bar{f}: \Sigma_{\bar{A}} \rightarrow \Sigma_{\bar{B}}$ which is right and left resolving and such that $f=h^{-1} \bar{f} g$.

It is easy to see that if $f$ is constant-to-one, of degree $d$, then $\bar{f}$ must be exactly $d$-to-one as a map on symbols.

2. Main results. In this section, we prove our main theorem, which should be compared with [B1, Theorem 2.2 and Corollary 2.4].

THEOREM 1. Let $\Sigma_{A}$ be an irreducible subshift of finite type, and $\lambda$ a nonzero eigenvalue for $A$. Then there is a finite set $E$ of positive integers such that if $\Sigma_{B}$ is an irreducible subshift of finite type, and $\lambda$ is an eigenvalue for $B$ which has the same multiplicity in $\chi_{A}$ and $\chi_{B}$, and if $f: \Sigma_{A} \rightarrow \Sigma_{B}$ is a constant-to-one factor map of degree $d$, then $d=e u$, where $e \in E$ and $u$ is a unit in $\mathbf{Z}[1 / \lambda]$.

COROLLARY. Let $f$ be a constant-to-one endomorphism of $\Sigma_{A}$ and $\lambda$ a nonzero eigenvalue. If $f$ has degree $d$, then $d$ is a unit in $\mathbf{Z}[1 / \lambda]$.

Later we will show that Theorem 1 is a consequence of the following:

THEOREM 2. Assume the same hypotheses as in Theorem 1. Let

$$
r=\left(r_{1}, \ldots, r_{n}\right)^{T}, \quad r_{i} \in \mathbf{Z}[1 / \lambda],
$$

be a right eigenvector for $A$, and $l=\left(l_{1}, \ldots, l_{n}\right), l_{i} \in \mathbf{Z}[1 / \lambda]$, a generalized left eigenvector (i.e. $l(A-\lambda I)^{s}=0$ for some s). If $f: \Sigma_{A} \rightarrow \Sigma_{B}$ is a constant-to-one factor map of degree $d$, then $d$ divides $l \cdot r$ in $\mathbf{Z}[1 / \lambda]$. 
REMARK. Such an $l$ and $r$ always exist-we get $l_{i}, r_{i} \in \mathbf{Q}[\lambda]$ by linear algebra, and by multiplying $l$ and $r$ by a sufficiently large integer, we force $l_{i}, r_{i}$ to lie in $\mathbf{Z}[\lambda] \subset \mathbf{Z}[1 / \lambda]$ (the last inclusion since $\chi_{A}$ is monic).

First we prove a lemma.

LEMMA. Let $l$ and $r$ be as in Theorem 2 , and let $\Sigma_{\bar{A}}$ be topologically conjugate to $\Sigma_{A}$. Then there exist a right eigenvector $r^{\prime}=\left(r_{1}^{\prime}, \ldots, r_{m}^{\prime}\right)^{T}, r_{i}^{\prime} \in \mathbf{Z}[1 / \lambda]$, and a generalized left eigenvector $l^{\prime}=\left(l_{1}^{\prime}, \ldots, l_{m}^{\prime}\right), l_{i}^{\prime} \in \mathbf{Z}[1 / \lambda]$, such that $l^{\prime} \cdot r^{\prime}=l \cdot r$.

Proof. Proceeding inductively, we first assume that $A$ and $\bar{A}$ are strong shift equivalent in one step (see [W1] for definitions), so that $A=U V, \bar{A}=V U$, for nonnegative, integral matrixes $U, V$. Let $r^{\prime}=V r$ and $l^{\prime}=l U / \lambda$. Then $l^{\prime} \cdot r^{\prime}=$ $l U / \lambda \cdot V r=l A r / \lambda=l \cdot r$. The proof for an arbitrary conjugate pair $\Sigma_{A}$ and $\Sigma_{\bar{A}}$ now follows by induction on the length of the strong shift equivalence between $A$ and $\bar{A}$.

ProOF OF THEOREM 2. We now assume that $f: \Sigma_{A} \rightarrow \Sigma_{B}$ is constant-to-one of degree $d$, and that $\bar{f}: \Sigma_{\bar{A}} \rightarrow \Sigma_{\bar{B}}$ is right and left resolving, with $\Sigma_{\bar{A}}$ conjugate to $\Sigma_{A}$ and $\Sigma_{\bar{B}}$ conjugate to $\Sigma_{B}$, as in Nasu's theorem. Let $l^{\prime}$ and $r^{\prime}$ be the vectors guaranteed by the previous lemma. We will show that $d$ divides $l^{\prime} \cdot r^{\prime}$ (and hence $l \cdot r)$ in $\mathbf{Z}[1 / \lambda]$.

Let $R$ be the relation matrix for $\bar{f}$, so that $\bar{A} R=R \bar{B}$ and $R^{T} \bar{A}=\bar{B} R^{T}$. If $\bar{A}$ is $m \times m$, and $\bar{B}$ is $k \times k$, then $R$ defines an injective linear map $L: \mathbf{R}^{k} \rightarrow \mathbf{R}^{m}$. The matrix equations tell us that the generalized right and left eigenspaces for $\bar{B}$ are carried into the generalized right and left eigenspaces for $\bar{A}$ respectively. Since conjugacy preserves the dimensions of generalized eigenspaces corresponding to nonzero eigenvalues (see [W2, Corollary 4.8]), the condition that $\lambda$ has the same multiplicity in $\chi_{A}$ as in $\chi_{B}$ implies that the $\bar{A}$ generalized eigenspaces for $\lambda$ have the same dimension as the $\bar{B}$ generalized eigenspaces, and so $L$ restricted to generalized eigenspaces is an isomorphism.

It follows from these remarks that $l^{\prime}$ and $r^{\prime}$ are in the image of $L$. Thus $r^{\prime}=R \beta$ where $\beta$ is a $\bar{B}$ generalized right eigenvector for $\lambda$. Since $r_{i}^{\prime}=\sum_{j=1}^{k} R_{i j} \beta_{j}$, it follows that $r_{i}^{\prime}=\beta_{j}$ if $\hat{f}(i)=j$ (where $\hat{f}$ is the induced map on vertices), so that the entries of $\beta$ lie in $\mathbf{Z}[1 / \lambda]$. Similarly, $l^{\prime}=\alpha R^{T}$, where $\alpha$ is a $\bar{B}$ generalized left eigenvector whose components lie in $\mathbf{Z}[1 / \lambda]$. Since $f$ is constant to one, $\bar{f}$ must be exactly $d$-to-one as a map on symbols, and it is easy to check that $R^{T} R=d I$. Thus $l^{\prime} \cdot r^{\prime}=\alpha R^{T} R \beta=d \alpha \cdot \beta$.

PROOF OF THEOREM 1. We now show that Theorem 1 follows from Theorem 2. First, we show that for any eigenvalue $\lambda$ for $A$, there exists a right eigenvector $r$ and a generalized left eigenvector $l$ (both corresponding to $\lambda$ and having components in $\mathbf{Z}[1 / \lambda])$ such that $l \cdot r \neq 0$. Assume that $A$ is $n \times n$, and let $r$ be a right eigenvector corresponding to $\lambda$. By the remark following Theorem 2, we may assume that the entries of $r$ lie in $\mathbf{Z}[1 / \lambda]$. If $l$ is a generalized left eigenvector corresponding to an eigenvalue $\gamma \neq \lambda$, then $l \cdot r=0$, by a standard argument. Since the generalized eigenvectors span all of $\mathbf{C}^{n}, r$ cannot be perpendicular to every generalized left eigenvector. If we choose a collection of generalized left eigenvectors, with components in $\mathbf{Q}[1 / \lambda]$, which span the generalized left eigenspace corresponding to $\lambda$, then at least one of these will have nonzero inner product with $r$. Again, we 
can multiply this vector by a sufficiently large integer so that its components lie in $\mathbf{Z}[1 / \lambda]$.

The remainder of the proof of Theorem 1 now follows exactly as in the proof of [B1, Theorem 2.2]. We repeat Boyle's argument here for completeness.

Let $l$ and $r$ be as above. Since $1 /(l \cdot r) \in \mathbf{Q}[1 / \lambda]$, there exists a positive integer $m$ such that $m /(l \cdot r) \in \mathbf{Z}[\lambda] \subset \mathbf{Z}[1 / \lambda]$. Let $E$ be the set of positive integers dividing $m$. If $f: \Sigma_{A} \rightarrow \Sigma_{B}$ is constant-to-one, of degree $d$, then $(l \cdot r) / d \in \mathbf{Z}[1 / \lambda]$, by Theorem 2. Thus

$$
\frac{m}{d}=\frac{m}{l \cdot r} \cdot \frac{l \cdot r}{d} \in \mathbf{Z}\left[\frac{1}{\lambda}\right] .
$$

Write $d=e u$, where $e=\operatorname{gcd}(m, d) \in E$. Let $k=m / e$. Then

$$
\frac{k}{u}=\frac{k e}{u e}=\frac{m}{d} \in \mathbf{Z}\left[\frac{1}{\lambda}\right],
$$

with $k$ and $u$ relatively prime. If $p$ is a prime dividing $u$, then

$$
\frac{k}{p}=\frac{u}{p} \cdot \frac{k}{u} \in \mathbf{Z}\left[\frac{1}{\lambda}\right]
$$

and therefore $1 / p \in \mathbf{Z}[1 / \lambda]$. It follows by multiplying that $1 / u \in \mathbf{Z}[1 / \lambda]$.

ProOF OF COROLLARY. To prove the corollary, given $\Sigma_{A}$, let $E$ be the finite set guaranteed by Theorem 1 . If $f$ is a constant-to-one endomorphism of $\Sigma_{A}$, of degree $d$, then $d=e u$, where $e \in E$ and $u$ is a unit in $\mathbf{Z}[1 / \lambda]$. Now $f^{k}$ is a constantto-one endomorphism, of degree $e^{k} u^{k}$. This forces some power of $e$, and therefore $e$ itself, to be a unit in $\mathbf{Z}[1 / \lambda]$. (See [B1, Corollary 2.4].)

We do not know whether, in Theorems 1, 2 or the corollary, the condition that $f$ be constant-to-one can be weakened to $d$-to-one almost everywhere. Nor do we know whether the condition on the multiplicity of $\lambda$ is necessary in Theorems 1 or 2.

EXAMPLE. Let

$$
A=\left(\begin{array}{lll}
0 & 1 & 1 \\
1 & 0 & 1 \\
1 & 1 & 0
\end{array}\right) .
$$

Then $\chi_{A}=(x-2)(x+1)^{2}$. Since the entropy of $\Sigma_{A}$ is $\log 2$, Boyle's theorem tells us only that any endomorphism $f$ of $\Sigma_{A}$ must have degree a power of 2 . If we assume that $f$ is constant-to-one, of degree $d$, then since $\lambda=-1$ is an eigenvalue and the only units in $\mathbf{Z}[1 / \lambda]=\mathbf{Z}$ are \pm 1 , by the corollary we must have $d=1$; thus $f$ must be an automorphism.

The rational units of $\mathbf{Z}[1 / \lambda]$ can be computed from the following proposition.

PROPOSITION. Let $\lambda$ be an algebraic integer with minimal polynomial $r(x)=$ $x^{n}+a_{n-1} x^{n-1}+\cdots+a_{0}$, and suppose $d \in \mathbf{Z}$. Then the following conditions are equivalent.

(1) $d$ is a unit in $\mathbf{Z}[1 / \lambda]$.

(2) If $p$ is a rational prime dividing $d$, then $p$ divides $a_{i}, 0 \leq i \leq n-1$.

(3) $d$ divides some power of the greatest common divisor of $\left\{a_{i}: 0 \leq i \leq n-1\right\}$.

ProOF. See [B1, Proposition 2.3]. Now, by the corollary, if $f$ is a constant-toone endomorphism of $\Sigma_{A}$, of degree $d$, then $d$ is a unit in $\mathbf{Z}[1 / \lambda]$ for all nonzero 
eigenvalues $\lambda$. By the above proposition, this implies that if $p$ is a rational prime dividing $d$, then $p$ divides all the nonleading coefficients of the characteristic polynomial of $A$.

M. Boyle has pointed out that there is a partial converse to the corollary to Theorem 1.

THEOREM (BOYLE, [B2]). Let $A$ be a nonnegative, integral matrix, whose minimal polynomial has degree $m$, and suppose that $d$ is a positive integer which is a unit in $\mathbf{Z}[1 / \lambda]$, for every nonzero eigenvalue $\lambda$ for $A$. If $n \geq m$, then there exists a constant-to-one endomorphism of $\Sigma_{A^{n}}$ of degree $d$.

ProOF. Suppose $p$ is a prime dividing $d$. Let $q(x)$ denote the minimal polynomial for $A$. Since $q(x)$ divides $\chi_{A}$, then by the preceding proposition, $p$ divides every nonleading coefficient of $q(x)$. Since $q(x)$ is monic, of degree $m$, and $q(A)=0$, it follows that $p$ divides every entry of $A^{n}$, for all $n \geq m$. Let $B=(1 / p) A^{n}$. Then $\Sigma_{A^{n}} \cong \Sigma_{B} \times \Sigma_{(p)}$, where $\Sigma_{(p)}$ is the full shift on $p$ symbols. It is well known that $\Sigma_{(p)}$ admits a constant-to-one endomorphism of degree $p$. For example, let $g$ be defined by the two block map $g\left(x_{i} x_{i+1}\right)=x_{i}+x_{i+1}(\bmod p)$, where the symbol set for $\Sigma_{(p)}$ is $\{0,1, \ldots, p-1\}$. Let $\tilde{f}: \Sigma_{B} \times \Sigma_{(p)} \rightarrow \Sigma_{B} \times \Sigma_{(p)}$ be defined by $\tilde{f}(x, y)=(x, g(y))$. Then $\tilde{f}$ is constant-to-one, of degree $p$, and $\tilde{f}$ extends naturally to a constant-to-one endomorphism $f_{p}$ of $\Sigma_{A^{n}}$, of degree $p$. Now the theorem follows by letting $f=f_{p_{1}}^{e_{1}} f_{p_{2}}^{e_{2}} \cdots f_{p_{k}}^{e_{k}}$ where $d=\prod_{i=1}^{k} p_{p_{i}}^{e_{i}}$ is the prime factorization of $d$.

\section{REFERENCES}

[A-M] R. L. Adler and B. Marcus, Topological entropy and equivalence of dynamical systems, Mem. Amer. Math. Soc., vol. 20, no. 219, 1979.

[B1] M. Boyle, Constraints on the degree of a sofic homomorphism and the induced multiplication of measures on unstable sets, Israel J. Math. 53 (1986), 52-68.

[B2] _ personal communication.

[H] G. A. Hedlund, Endomorphisms and automorphisms of the shift dynamical system, Math. Systems Theory 3 (1969), 320-375.

[K] B. Kitchens, Continuity properties of factor maps in ergodic theory, Ph.D. thesis, Univ. of North Carolina, Chapel Hill, 1981.

[N] M. Nasu, Constant-to-one and onto global maps of homomorphisms between strongly connected graphs, Ergodic Theory Dynamical Systems 3 (1983), 387-414.

[P-T] W. Parry and S. Tuncel, Classification problems in ergodic theory, London Math. Society Lecture Note Series 67, Cambridge Univ. Press, 1982.

[W1] R. Williams, Classification of subshifts of finite type, Ann. of Math. (2) 98 (1973), 120-153.

[W2] , Classification of one-dimensional attractors, Proc. Sympos. Pure Math., vol. 14, Amer. Math. Soc., Providence, R.I., 1970, pp. 341-361.

Department of Mathematics, NORThWESTERn UNiVERSity, EVANSton, ILlinois 60201

Current address: Department of Mathematics, University of California, Berkeley, California 94720 